Pacific Journal of Mathematics

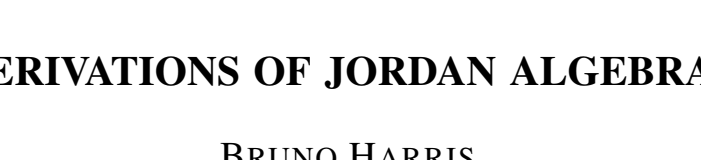




\title{
DERIVATIONS OF JORDAN ALGEBRAS
}

\author{
BRUNO HARRIS
}

Introduction. Let $J$ be a finite-dimensional semisimple Jordan algebra over a field of characteristic zero, and $D$ a derivation of $J$ into a module $M$. Jacobson has shown, in [6], that $D$ is inner in the sense that there exist elements $z_{i}$ in $J, m_{i}$ in $M$ such that for all $x$ in $J$,

$$
D(x)=\sum_{i}\left(z_{i}, x, m_{i}\right)
$$

(where $\left(z_{i}, x, m_{i}\right)$ denotes the associator $\left(z_{i} \cdot x\right) \cdot m_{i}-z_{i} \cdot\left(x \cdot m_{i}\right)$ and $x \cdot y$ denotes the product in $J$ or the product of an element of $J$ and one of $M)$. This theorem is the analogue for Jordan algebras of the first Whitehead lemma for semisimple Lie algebras of characteristic zero. In this paper we will consider two problems: first to generalize the above theorem to arbitrary characteristic $p$ (excluding $p=2$ but allowing $p=3)$; second, to express the group of derivations modulo inner derivations of any Jordan algebra (not necessarily finite-dimensional or semisimple) as a cohomology group. The second problem is part of a much more general one: that of developing a cohomology theory for Jordan algebras analogous to the existing theories for associative and Lie algebras (see [9]).

Our results are as follows: with respect to the first problem, we show that if $J$ is finite-dimensional and separable, then every derivation of $J$ into a module is inner (i.e. satisfies (1)) if and only if $J$ satisfies the additional condition that it has no simple ideal which is special and whose degree is divisible by the characteristic of the base field. (This latter condition is directly related to the fact that the Lie algebra of all $n \times n$ matrices over a field of characteristic $p$ cannot be expressed as the direct sum of the derived algebra and the center if $p$ divides $n$.) For the proof we use the representation theory of Jordan algebras given in [7] and rely to a certain extent on the classification of simple algebras; however, it may be possible to give a proof not relying on the classification by using a Casimir operator (as is done for Lie and alternative algebras in [3]).

As for the second problem, our results cover only special Jordan algebras and certain types of modules. We use the concept of a bimodule with involution (introduced in [7]) for an associative algebra with involution, and introduce cohomology groups which are like the usual cohomology groups of associative algebras but also take into account

Received September 22, 1958. This work was supported in part by a grant from the National Science Foundation. 
the involution. These cohomology groups may be of interest in themselves, but in this paper we examine only the first cohomology group and its relation to derivations of special Jordan algebras.

Together with the Jordan results we obtain the first Whitehead lemma for separable alternative algebras of characteristic not 2 . This result has been proved for characteristic $\neq 2,3$ by Taft [13], using only the alternative structure theory; previously it has been proved for characteristic zero by Schafer [12] using the result on Jordan algebras. A Casimir operator method is given by Campbell, [3], who does not specify for what characteristic his assumptions are fulfilled. By using an appropriate definition of inner derivations we are able to include characteristic 3 (in [10] Kaplansky announced that the Cayley numbers have outer derivations in characteristic 3 , but presumably he used a different definition of inner derivations).

We are indebted to Professor Jacobson and Dr. Taft for conversations on these problems. The derivations of some of the simple Jordan algebras have been worked out independently by them.

Preliminaries. We will consider Jordan algebras over fields of characteristic different from 2. The product in the Jordan algebra, denoted by $x \cdot y$, satisfies

$$
\begin{gathered}
x \cdot y=y \cdot x \\
((x \cdot x) \cdot y) \cdot x=(x \cdot x) \cdot(y \cdot x) .
\end{gathered}
$$

A module $M$ for $J$ is a vector space with a bilinear composition $x \cdot m$ for $x$ in $J, m$ in $M$ such that the vector space direct sum (or "split null extension") $E=J \oplus M$ with the multiplication $(x+m) \cdot(y+n)=$ $(x \cdot y+x \cdot n+y \cdot m)$ is a Jordan algebra, i.e. the product satisfies (2) and (3). In [7] a slightly different definition of module is given: namely, a multilinear identity is used instead of (3); the multilinear identity is implied by (3) for characteristic not 2 and is equivalent to (3) for characteristic not 2 or 3 . With the present definition the results of [7] are valid for characteristic 3 also (cf. the footnote on p. 5 of [7], which however should read "... implies the original identity if the characteristic is $\neq 3$ " and also footnote $3, \mathrm{p} .18$, of [9]). We refer to [7] for all the concepts and results on the module theory of Jordan algebras that we shall use.

A module $M$ is called special if

$$
(x \cdot y) \cdot m=x \cdot(y \cdot m)+y \cdot(x \cdot m) \text { for } x, y \text { in } J, m \text { in } M .
$$

Correspondingly we define a universal associative algebra $U^{\prime}$ such that every special $J$ module is a left module for the associative algebra $U^{\prime}$. To each element $x$ of $J$ corresponds an element $x^{\prime}$ of $U^{\prime}$ such that 
$x \cdot m=x^{\prime} m$ if $M$ is a special module. If the $\operatorname{map} x \rightarrow x^{\prime}$ is one-to-one we call $J$ a special Jordan algebra; in this case we will write $x$ for $x^{\prime}$ and consider $J$ as a Jordan subalgebra of $U^{\prime}$ with product $x \cdot y=x y+y x$. $U^{\prime}$ is generated by the identity and by the elements $x^{\prime}$, and has an involution which is the identity on the $x^{\prime}$.

A module $M$ for $J$ is called a submodule of a sum of commuting special bimodules if there is an associative bimodule (i.e. two-sided module) $N$ for $U^{\prime}$ such that $M$ is a subspace of $N$ and the composition in $M$ is

$$
x \cdot m=x^{\prime} m+m x^{\prime} .
$$

If $J$ is special, a module $M$ satisfies this condition if and only if $E=$ $J \oplus M$ is also a special Jordan algebra. The universal associative algebra for such modules is denoted by $U^{\prime \prime}$, and we shall call $M$ a $U^{\prime \prime}$ module. Every special module $M$ can be considered a $U^{\prime \prime}$ module as follows: Let $N$ be the vector space direct sum $M \oplus M$ of $M$ with itself. Define $N$ as $U^{\prime}$ bimodule by: $x^{\prime}(a \oplus b)=\left(x^{\prime} a\right) \oplus 0,(a \oplus b) x^{\prime}=0 \oplus\left(x^{\prime} b\right)$, for $x \in J$, then $M$ is isomorphic to the subspace of elements $m \oplus m$ of $N$. Using the involution $a \rightarrow a^{*}$ in $U^{\prime}$, we can speak of left $U^{\prime} \otimes U^{\prime}$ (tensor product over the base field) modules $N$, instead of $U^{\prime}$ bimodules; let $(a \otimes b) n=a n b^{*}$ for $n \in N$. Then $U^{\prime \prime}$ is isomorphic to the subalgebra of $U^{\prime} \otimes U^{\prime}$ generated by $1 \otimes 1$ and all $x^{\prime} \otimes 1+1 \otimes x^{\prime}$ for $x$ in $J$.

A derivation of a Jordan algebra $J$ into a module $M$ is a linear map $D$ of $J$ into $M$ satisfying

$$
D(x \cdot y)=x \cdot D(y)+y \cdot D(x) .
$$

A definition of inner derivation of any non-associative algebra (into itself) was given by Schafer [11]: a derivation is inner if it belongs to the Lie algebra of linear transformations on the algebra generated by the right and left multiplications. If we extend this definition to derivations into modules (using the extension $E=J \oplus M$ ), and if we consider Jordan algebras with identity elements, then an inner derivation in this sense has the form (1), and conversely any map of the form (1) is inner. If $J$ is special and $M$ is a $U^{\prime \prime}$ module, then, on using (5), (1) becomes

$$
D(x)=\left[x, \sum_{i}\left[z_{i}, m_{i}\right]\right]
$$

where $[a, b]$ denotes $a b-b a$. (7) suggests the following more general definition of inner derivation: if $J$ is a special module, $M$ a $U^{\prime \prime}$ module contained in a $U^{\prime}$-bimodule $N$, then a derivation is inner if it has the form

$$
D(x)=[x, n]
$$

for $n$ in $N$. 
Thus a derivation of the form (8) is also of form (1) (or (7)) if $n=$ $\sum_{i}\left[z_{i}, m_{i}\right]$ for elements $z_{i}$ in $J, m_{i}$ in $M$.

If a module $M$ is a direct sum of two submodules $M_{1}$ and $M_{2}$ and $D$ is a derivation of $J$ into $M$, then on writing

$$
D(x)=D_{1}(x)+D_{2}(x), D_{i}(x) \in M_{i},
$$

the $D_{i}$ are derivations and are inner in the sense of (1) if and only if $D$ is inner in the sense of (1). Similar remarks apply to inner derivations of the form (8).

\section{Derivations of Special Jordan Algebras}

In this section we will consider a situation illustrated by the following example: $J$ is the set of self-adjoint elements of an associative algebra $A$ with involution, and $M$ is $J$ itself. It is natural to call a derivation inner if it is of the form $x \rightarrow[x, a]$ where $a$ is a skew element of $A$. We wish to describe the group of derivations mod inner ones as a cohomology group.

We begin with a general discussion of associative algebras and bimodules with involution (see [7], p. 40) and introduce cohomology groups for them (see [4]).

If $A$ is an associative algebra with identity 1 and involution $a \rightarrow a^{*}$ and $N$ is an associative bimodule for $A, N$ is called a bimodule with involution if it possesses a linear transformation $n \rightarrow \bar{n}$ such that

$$
\overline{\bar{n}}=n, \quad \overline{a n}=\bar{n} a^{*}, \quad \overline{n a}=a^{*} \bar{n} \text { for } a \in A, n \in N .
$$

$N$ may also be considered as left $A \otimes A$ module by $(a \otimes b) n=a n b^{*}$, in which case

$$
(\overline{a \otimes b) n}=(b \otimes a) \bar{n}, \text { and } \overline{\bar{n}}=n .
$$

A bimodule with involution can also be described as a left module over an algebra $K$ constructed as follows: in $A \otimes A$ consider the automorphism $\sigma: a \otimes b \rightarrow b \otimes a$, and let $K$ be the crossed product algebra consisting of all pairs $(\alpha, \beta)$ with $\alpha, \beta \in A \otimes A$, with componentwise addition, and multiplication $(\alpha, \beta)(\gamma, \delta)=\left(\alpha \gamma+\beta \delta^{\sigma}, \alpha \delta+\beta \gamma^{\sigma}\right)$ where $\alpha \rightarrow \alpha^{\sigma}$ is the automorphism just described. We can also represent $K$ as the set of all elements of the form $\alpha+\beta \sigma, \alpha, \beta$ in $A \otimes A$, with multiplication rules $\sigma \alpha=\alpha^{\sigma} \sigma, \sigma^{2}=1$. If $N$ is a bimodule with involution, setting $\sigma n=\bar{n}$ makes $N$ a left $K$ module, and conversely if $N$ is a left $K$ module, the map $n \rightarrow \bar{n}=\sigma n$ makes $N$ a bimodule with involution.

$A$ itself is a left $A \otimes A$ module under $(a \otimes b) c=a c b^{*}$. It can be made a bimodule with involution in two ways: either by letting $\bar{a}=a^{*}$, or by letting $\bar{a}=-a^{*}$. 
We define the $n$th cohomology group (see [4]) as $\operatorname{Ext}_{K}^{n}(A, N)$ where we consider $A$ as left $K$ module with $\sigma a=-a^{*}$. We shall now exhibit a specific projective resolution of $A$ as $K$ module.

\section{Let}

$$
\begin{aligned}
& X_{0}=K(1-\sigma), \\
& \quad X_{n}=\left\{\sum \alpha \otimes a_{1} \otimes \cdots \otimes a_{n}+\omega_{n} \alpha \sigma \otimes a_{n}^{*} \otimes \cdots \otimes a_{1}^{*} \mid \alpha \in A \otimes A \subseteq K, a_{i} \in A_{j}\right.
\end{aligned}
$$

for $n \geqq 0$, where $\omega_{n}=(-1)^{\frac{1}{2}(n-1)(n-2)}$.

Setting

$$
e=\frac{1+\sigma}{2}, f=\frac{1-\sigma}{2},
$$

we see that $X_{n}=K e \otimes H_{n} \oplus K f \otimes S_{n}$ where $H_{n}$ is the subspace of $A_{n}=A \otimes \cdots \otimes A$ ( $n$ factors) of elements $x$ such that $T x=x$ and $S_{n}$ is the subspace of $x$ such that $T x=-x$, where $T\left(a_{1} \otimes \cdots \otimes a_{n}\right)=$ $(-1)^{\frac{1}{2}(n-1)(n-2)} a_{n}^{*} \otimes \cdots \otimes a_{1}^{*}$. We make $X_{n}$ a left $K$ module by defining $k\left(k_{1} e \otimes h_{n}+k_{2} f \otimes s_{n}\right)=k k_{1} e \otimes h_{n}+k k_{2} f \otimes s_{n}$ for $k \in K$. Since $e, f$ are idempotents, $X_{n}$ is $K$-projective.

We define $K$-homomorphisms $d_{n}: X_{n} \rightarrow X_{n-1}$ as follows: we first define a map $d_{n}: K \otimes A_{n} \rightarrow K \otimes A_{n-1}$ by

$$
\begin{aligned}
d_{n}\left(k \otimes a_{1} \otimes \cdots \otimes a_{n}\right)= & k\left(a_{1} \otimes 1\right) \otimes a_{2} \otimes \cdots \otimes a_{n} \\
& +\sum_{i=1}^{n-1}(-1)^{i} k \otimes a_{1} \otimes \cdots \otimes a_{i} a_{i+1} \otimes \cdots \otimes a_{n} \\
& +(-1)^{n} k\left(1 \otimes a_{n}^{*}\right) \otimes a_{1} \otimes \cdots \otimes a_{n-1}
\end{aligned}
$$

and note that $d_{n-1} \cdot d_{n}=0$. Next we verify that $d_{n}$ maps $X_{n} \rightarrow X_{n-1}$ :

$$
\begin{aligned}
& d_{n}\left(\alpha \otimes a_{1} \otimes \cdots \otimes a_{n}+\omega_{n} \alpha \sigma \otimes a_{n}^{*} \otimes \cdots \otimes a_{1}^{*}\right) \\
& =\alpha\left(a_{1} \otimes 1\right) \otimes a_{2} \otimes \cdots \otimes a_{n}+\sum_{i=1}^{n-1}(-1)^{i} \alpha \otimes a_{1} \otimes \cdots \otimes a_{i} a_{i+1} \otimes \cdots \otimes a_{n} \\
& \quad+(-1)^{n} \alpha\left(1 \otimes a_{n}^{*}\right) \otimes a_{1} \otimes \cdots \otimes a_{n-1}+\omega_{n} \alpha \sigma\left(a_{n}^{*} \otimes 1\right) \otimes a_{n-1}^{*} \otimes \cdots \otimes a_{1}^{*} \\
& \quad+\sum_{i=1}^{n-1}(-1)^{n-i} \omega_{n} \alpha \sigma \otimes a_{n}^{*} \otimes \cdots \otimes a_{i+1}^{*} a_{i}^{*} \otimes \cdots \otimes a_{1}^{*} \\
& \quad+(-1)^{n} \omega_{n} \alpha \sigma\left(1 \otimes a_{1}\right) \otimes a_{n}^{*} \otimes \cdots \otimes a_{2}^{*} . \\
& =\left[\alpha\left(a_{1} \otimes 1\right)\right] \otimes a_{2} \otimes \cdots \otimes a_{n}+(-1)^{n} \omega_{n}\left[\alpha\left(a_{1} \otimes 1\right)\right] \sigma \otimes a_{n}^{*} \otimes \cdots \otimes a_{2}^{*} \\
& \quad+\sum_{i=1}^{n-1}(-1)^{i}\left(\alpha \otimes a_{1} \otimes \cdots \otimes a_{i} a_{i+1} \otimes \cdots \otimes a_{n}\right. \\
& \left.\quad+(-1)^{n} \omega_{n} \alpha \sigma \otimes a_{n}^{*} \otimes \cdots \otimes\left(a_{i} a_{i+1}\right)^{*} \otimes \cdots \otimes a_{1}^{*}\right) \\
& \quad+(-1)^{n}\left(\alpha\left(1 \otimes a_{n}^{*}\right) \otimes a_{1} \otimes \cdots \otimes a_{n-1}\right. \\
& \left.\quad+(-1)^{n} \omega_{n} \alpha\left(1 \otimes a_{n}^{*}\right) \sigma \otimes a_{n-1}^{*} \otimes \cdots \otimes a_{1}^{*}\right) .
\end{aligned}
$$

Since

$$
(-1)^{n} \omega_{n}=(-1)^{n}(-1)^{\frac{1}{2}(n-1)(n-2)}=(-1)^{\frac{1}{2}\left(n^{2}-n+2\right)}=(-1)^{\frac{1}{2}\left(n^{2}-5 n+6\right)}=\omega_{n-1}
$$


we see that the last term is indeed in $X_{n-1}$.

We also define $\varepsilon: X_{0} \rightarrow A$ by $(a \otimes b)\left(\frac{1}{2}(1-\sigma)\right) \rightarrow a b^{*}$. Then $\varepsilon$ and the $d_{n}$ are all $k$-homomorphisms, and $\varepsilon \cdot d_{1}=0$.

Finally, we have to show the sequence

$\cdots \rightarrow X_{n} \stackrel{d_{n}}{\longrightarrow} X_{n-1} \longrightarrow \cdots \stackrel{d_{1}}{\longrightarrow} X_{0} \stackrel{\varepsilon}{\longrightarrow} A \longrightarrow 0$ is exact. We use the fact that the corresponding sequence used to define the usual homology groups for $A$ (i.e. using $(A \otimes A) \otimes A_{n}$ instead of $\left.X_{n}\right)$ is exact, and that $\alpha_{1}+\alpha_{2} \sigma=0$ in $K$ for $\alpha_{1}, \alpha_{2}$ in $A \otimes A$ implies $\alpha_{1}=0=\alpha_{2}$. Let now

$$
d_{n}\left(\alpha \otimes a_{1} \otimes \cdots \otimes a_{n}+\omega_{n} \alpha \sigma \otimes a_{n}^{*} \otimes \cdots \otimes a_{1}^{*}\right)=0 .
$$

Then $d_{n}\left(\alpha \otimes a_{1} \otimes \cdots \otimes a_{n}\right)=0$ in $(A \otimes A) \otimes A_{n-1}$, and so

$$
\begin{aligned}
\alpha \otimes a_{1} \otimes \cdots \otimes a_{n}= & d_{n+1}\left(\sum \beta \otimes b_{1} \otimes \cdots \otimes b_{n+1}\right) \\
= & \sum\left(\beta\left(b_{1} \otimes 1\right) \otimes b_{2} \otimes \cdots \otimes b_{n+1}\right. \\
& +\sum_{i=1}^{n}(-1)^{i} \beta \otimes b_{i} \otimes \cdots \otimes b_{i} b_{i+1} \otimes \cdots \otimes b_{n+1} \\
& \left.+(-1)^{n+1} \beta\left(1 \otimes b_{n+1}^{*}\right) \otimes b_{1} \otimes \cdots \otimes b_{n}\right)
\end{aligned}
$$

therefore

$$
\begin{aligned}
\alpha \sigma \otimes a_{n}^{*} \otimes \cdots \otimes a_{1}^{*}= & \sum\left(\beta\left(b_{1} \otimes 1\right) \sigma \otimes b_{n+1}^{*} \otimes \cdots \otimes b_{2}^{*}\right. \\
& +\sum_{i=1}^{n}(-1)^{i} \beta \sigma \otimes b_{n+1}^{*} \otimes \cdots \otimes b_{i+1}^{*} b_{1}^{*} \otimes \cdots \otimes b_{1}^{*} \\
& \left.+(-1)^{n+1} \beta\left(1 \otimes b_{n+1}^{*}\right) \sigma \otimes b_{n}^{*} \otimes \cdots \otimes b_{1}^{*}\right) \\
= & (-1)^{n+1} d_{n+1}\left(\sum \beta \sigma \otimes b_{n+1}^{*} \otimes \cdots \otimes b_{1}^{*}\right) .
\end{aligned}
$$

Thus $\alpha \otimes a_{1} \otimes \cdots \otimes a_{n}+\omega_{n} \alpha \sigma \otimes a_{n}^{*} \otimes \cdots \otimes a_{1}^{*}$

$$
=d_{n+1}\left(\sum \beta \otimes b_{1} \otimes \cdots \otimes b_{n+1}+(-1)^{n+1} \omega_{n} \beta \sigma \otimes b_{m+1}^{*} \otimes \cdots \otimes b_{1}^{*}\right)
$$

but $(-1)^{n+1} \omega_{n}=\omega_{n+1}$, and so we have shown that kernel of $d_{n}=$ image of $d_{n+1}$ and in exactly the same way we see that kernel of $\varepsilon=$ image of $d_{1}$. It is clear that the image of $\varepsilon$ is $A$. Thus we have a projective resolution of $A$.

If $N$ is a left $K$ module, the vector space $\operatorname{Hom}_{K}\left(X_{n}, N\right)$ can be identified with the space of linear function $g$ on $A_{n}$ to $N$ which satisfy the condition $\sigma g\left(a_{1}, \cdots, a_{n}\right)=\omega_{n} g\left(a_{n}^{*}, \cdots, a_{1}^{*}\right)$, by identifying a $K$ homomorphism $h$ of $X_{n}$ into $K$ and the linear function

$$
g\left(a_{1}, \cdots, a_{n}\right)=h\left(1 \otimes a_{1} \otimes \cdots \otimes a_{n}+\omega_{n} \sigma \otimes a_{n}^{*} \otimes \cdots a_{1}^{*}\right) .
$$

The functions $g$ which correspond to cocycles or coboundaries are then cocycles or coboundaries in the usual sense which also satisfy the additional condition above.

In particular the 1-cocycles are functions $g$ on $A$ to $N$ which satisfy $g(a b)=a g(b)+g(a) b$ and $\left.g\left(a^{*}\right)=\overline{g(a}\right)$ i.e. derivations mapping self-adjoint 
elements of $A$ into self-adjoint elements of $N$, and skew elements into skew elements; the 1-coboundaries are functions of the form $g(a)=[a, n]$ where $n \in f N=$ skew elements of $N$.

We will now apply these considerations to the algebra $U^{\prime}$ of a special Jordan algebra $J$. (Actually everything we will do applies to any Jordan algebra $J$, provided we replace $J$ by its image in $U^{\prime}$, which is a homomorphic image of $J$ ). $U^{\prime}$ has an involution $a \rightarrow a^{*}$ such that $x=x^{*}$ for $x$ in $J$. We form the algebra $K$ as above, taking $U^{\prime}$ for $A$, and let $\sigma a=-a^{*}$ for $a \in U^{\prime}$. Our theorem is:

Theorem 1. Let $J$ be a special Jordan algebra, $M$ a $U^{\prime \prime}$-module such that $M=H(N)$, the set of self-adjoint elements of $N$, where $N i s$ an associative bimodule with involution for $U^{\prime}$. Then $\operatorname{Ext}_{K}^{1}\left(U^{\prime}, N\right)$ is isomorphic to the vector space of derivations of $J$ into $M$ modulo inner derivations of the form $x \rightarrow[x, n]$ where $n$ is a skew element of $N$.

Proof. We have to show that the derivations $D$ of $J$ into $M$ may be identified with the derivations $D_{0}$ of $U^{\prime}$ into $N$ which satisfy $D_{0} \sigma=$ $-\sigma D_{0}$ : Let $D$ be a derivation of $J$ into $M$. For $x$ in $J$, the map $h$ : $x \rightarrow x+D(x)$ of $J$ into $E=J \oplus M$ is a homomorphism (actually an isomorphism) of Jordan algebras. $E$ may be considered as a Jordan subalgebra of $B=U^{\prime} \oplus N$, and so $h$ can be extended to a homomorphism, also denoted by $h$, of $U^{\prime}$ into $B$ taking $1 \rightarrow 1$. For $a$ in $U^{\prime}, h(a)-a$ lies in $N$, since this is so for $a \in F_{1} \oplus J$ which is a set of generators for $U^{\prime}$ and since $N$ is an ideal in $B$. The map $D_{0}: a \rightarrow h(\alpha)-a$ is a derivation of $U^{\prime}$ into $N$, and $D_{0}(x)=D(x)$ for $x$ in $J . \quad D_{0}(\sigma a)=-\sigma D_{0}(a)$ since this equation holds for $a \in F 1 \oplus J$. The map $D \rightarrow D_{0}$ is clearly one-toone, since $D$ and $D_{0}$ coincide on a set of generators for $U^{\prime}$, and it is onto the vector space of derivations $D_{0}$ satisfying $D_{0} \sigma=-\sigma D_{0}$ since the restriction of such a $D_{0}$ to $J$ is a derivation $D$ of $J$ into $M$. Under this identification, the inner derivations $D_{0}$ of $U^{\prime}$ clearly correspond to inner derivations $D$ of $J$.

The above theorem applies only to a certain type of module for a special Jordan algebra. However, there exists a large class of special Jordan algebras for which all modules satisfy the conditions of the theorem, namely the Jordan algebras of all $n \times n$ hermitian matrices (with $n \geqq 4$ ) over an arbitrary involutorial associative algebra with identity (this is explicitly stated in [7], Th. 10.2, 10.4, 7.1 for unital modules; however every module for a Jordan algebra with identity is a direct sum of a unital module and a special module, and special modules also satisfy the condition, by the construction in the previous section). This class of Jordan algebras includes the algebras of all $n \times n$ matrices; the algebra of hermitian bounded operators (or of all bounded operators) on 
a Hilbert space, or more generally the hermitian operators (or all operators) in a factor, also belongs to the class. For these algebras of matrices, consideration of modules for the Jordan algebra can de reduced to consideration of bimodules with involution for the coefficient algebras ([7]), however in our theorem the hypotheses are much more general than this.

\section{Derivations of Separable Jordan and Alternative Algebras}

In this section we will consider finite dimensional separable Jordan and alternative algebras over fields of characteristic not 2. (A separable algebra is one which is semisimple and remains semisimple under any extension of the base field.) We will obtain the first Whitehead lemma for both classes of algebras as follows: first we will prove it for simple Jordan algebras of degree two, then for alternative algebras, and lastly for the other Jordan algebras.

We shall need the following facts on the structure of these algebras ([1], [2]): a simple Jordan algebra over an algebraically closed field is said to be of degree $n$ if the identity element is a sum of $n$, but not more, mutually orthogonal idempotents. A simple Jordan algebra over any field will be defined to be of degree $n$ if on extending its center to its algebraic closure it becomes an algebra of degree $n$. Over an algebraically closed field, the simple algebras are as follows: An algebra of degree one is just the base field, [(8]); all derivations are zero. An algebra of degree 2 is the vector space direct sum $F 1+V$ of the space $F 1$ spanned by its identity element 1 and a vector space $V$ of dimension at least two on which is defined a non-degenerate symmetric bilinear form $(x, y)$ and the multiplication in the algebra is

$$
(\alpha 1+x) \cdot(\beta 1+y)=[\alpha \beta+(x, y)] 1+(\alpha y+\beta x)
$$

for $\alpha, \beta \in F, x, y \in V$. An algebra of degree $n \geq 3$ consists of all $n \times n$ hermitian matrices with coefficients in an involutorial alternative algebra $D$ which has no proper self-adjoint ideals. $D$ is thus $F, E, Q$ or $C$ where $F$ is the base field, $E$ is two dimensional over $F, Q$ and $C$ are the quaternion and Cayley algebra respectively. $J$ is denoted by $H\left(D_{n}\right)$; if $D$ is $C$ then $n=3$. The Cayley algebra $C$ is generated by $Q$ and one other element $v$ with $v^{2}=\alpha 1, \alpha$ in $F$ (and $\alpha \neq 0$ ), $C=Q+Q v$ (vector space direct sum) and the multiplication is

$$
\left(q_{1}+q_{2} v\right)\left(q_{3}+q_{4} v\right)=\left(q_{1} q_{3}+\alpha \bar{q}_{4} q_{2}\right)+\left(q_{4} q_{1}+q_{2} \bar{q}_{3}\right) v .
$$

Our results are as follows:

Theorem 2. Let $J$ be a finite dimensional separable Jordan algebra. Then every derivation $D$ of $J$ into a module $M$ is inner, in the sense 
that there exist elements $z_{i}$ in $J$ and $m_{i}$ in $M$ such that $D(x)=\sum_{i}\left(z_{i} x, m_{i}\right)$ if and only if $J$ contains no simple ideal which is special and whose degree is divisible by the characteristic of the base field.

THeOREM 3. Let $A$ be a finite dimensional separable alternative algebra, $M$ a bimodule, and $D$ a derivation of $A$ into $M$. Then there exist elements $a_{i}$ in $A, m_{i}$ in $M$, and $g$ in the nucleus of $M$, such that

$$
D(x)=[x, g]+\sum_{i}\left[a_{i}, x, m_{i}\right] \text { and } \sum_{i}\left[a_{i}, m_{i}\right]=0
$$

where $[a, b, c]$ is the associator $(a b) c-a(b c)$. If the characteristic is not two or three, then

$$
D(x)=[x, g]+\sum_{i} D_{a_{i}, m_{i}}(x)
$$

$a_{i}$ in $A, m_{i}$ in $M, g$ in the nucleus of $M$, where

$$
D_{a, b}(x)=(x a) b-(x b) a+(a x) b-a(x b)+b(a x)-a(b x) .
$$

We remark that in any Jordan the map $x \rightarrow \sum\left(z_{i}, x, m_{i}\right)$ is a derivation, and in any alternative algebra the maps $x \rightarrow[x, g]$ with $g$ in the nucleus, $x \rightarrow \sum_{i} D_{a_{i}, m_{i}}(x)$, and $x \rightarrow \sum\left[a_{i}, x, m_{i}\right]$ with $\sum\left[a_{i}, m_{i}\right]=0$ are derivations if the characteristic is not two (see [12], formulas (12), (19)).

The proof of the theorems is in several steps:

(a) Let $J$ be a semi-simple Jordan algebra over an algebraically closed field, which satisfies the condition of the theorem concerning degrees. We want to show a derivation $D$ of $J$ into a module $M$ is of the form (1). A module $M$ is called unital if $\mathrm{e} \cdot m=m$ for all $m$ in $M$, where $e$ is the identity element of $J$. We first show:

1. $M$ may be assumed unital. In general, $M=M_{1}+M_{1 / 2}+M_{0}$ (direct sum) where $e \cdot m_{i}=i m_{i}$ for $m_{i}$ in $M_{i}, M_{1}$ is unital, and $M_{1 / 2}+M_{0}$ is a special module. Let $D(x)=D_{1}(x)+D_{2}(x), D_{1}(x) \in M_{1}, D_{2}(x) \in M_{1 / 2}+M_{0}$. Then $D_{1}, D_{2}$ are also derivations, and are of the form (1) if and only if $D$ is. Since we are assuming the result for $D_{1}$, we have to prove it for $D_{2}$. However, it is true, more generally, that if $J$ is any Jordan algebra with identity $e$ and $N$ is a special module then any derivation $D$ is of form (1): Let $N=N_{1 / 2}+N_{0}$, then $D=D_{1 / 2}+D_{0}$, and $D_{0}(x)=$ $D_{0}(e \cdot x)=e \cdot D_{0}(x)+x \cdot D_{0}(e)=0$ so $D$ maps $J$ into $N_{1 / 2}$.

$$
D(x)=D(e \cdot x)=e \cdot D(x)+x \cdot D(e)=\frac{1}{2} D(x)+x \cdot D(e),
$$

or $D(x)=2 x \cdot D(e)=4(e, x, D(e))$. 
2. $J$ may be assumed simple. Let $J=J_{1} \oplus \cdots \oplus J_{m}, J_{i}$ simple ideals satisfying the condition on degrees, and let $M$ be a unital $J$ module. Let $e_{i}$ be the identity of $J_{i}$, then $M$ decomposes into a direct sum of subspaces $M_{i i}, 1 \leqq i \leqq m$, and $M_{i j}=M_{j i}, i \neq j, 1 \leqq i, j \leqq m$, where $M_{i i}=$ $\left\{m \in M / e_{i} \cdot m=m\right\}$ and $M_{i j}=\left\{m \in M / e_{i} \cdot m=\frac{1}{2} m=e_{j} \cdot m\right\}$ for $i \neq j$.

Then $M_{i i}$ is unital for $J_{i}$ and annihilated by $J_{j}$ for $j \neq i, M_{i j}$ is special for $J_{i}$ and $J_{j}$ for $i \neq j$ and $x_{i} \cdot\left(x_{j} \cdot m_{i j}\right)=x_{j} \cdot\left(x_{i} \cdot m_{i j}\right)$ for

$$
x_{i} \in J_{i}, x_{j} \in J_{j}, m_{i j} \in M_{i j},
$$

and $M_{i j}$ is annihilated by $J_{k}$ for $k \neq i, j$.

Let $D$ be a derivation of $J$ into $M$. We want to show $D$ satisfies (1), assuming this for $J$ simple. Let $D(x)=\sum_{i} D_{i i}(x)+\sum_{i<j} D_{i j}(x)$ for $x \in J$, where $D_{i i}(x) \in M_{i i}, D_{i j}(x) \in M_{i j}$. For $x_{k}, y_{k} \in J_{k}$,

$$
\begin{aligned}
D\left(x_{k} \cdot y_{k}\right)=\sum_{i} D_{i i}\left(x_{k} \cdot y_{k}\right)+ & \sum_{i<j} D_{i j}\left(x_{k} \cdot y_{k}\right) \\
=x_{k} \cdot D\left(y_{k}\right)+y_{k} \cdot D\left(x_{k}\right)= & x_{k} \cdot\left(\sum_{i} D_{i i}\left(y_{k}\right)+\sum_{i<j} D_{i j}\left(y_{k}\right)\right) \\
& +y_{k} \cdot\left(\sum_{i} D_{i i}\left(x_{k}\right)+\sum_{i<j} D_{i j}\left(x_{k}\right)\right) \\
=x_{k} \cdot D_{k k}\left(y_{k}\right)+y_{k} \cdot D_{k k}\left(x_{k}\right) & +x_{k} \cdot\left(\sum_{i<k} D_{i k}\left(y_{k}\right)+\sum_{k<j} D_{k j}\left(y_{k}\right)\right) \\
& +y_{k} \cdot\left(\sum_{i<k} D_{i k}\left(x_{k}\right)+\sum_{k<j} D_{k j}\left(y_{k}\right)\right) .
\end{aligned}
$$

Thus $D_{k k}\left(x_{k} \cdot y_{k}\right)=x_{k} \cdot D_{k k}\left(y_{k}\right)+y_{k} \cdot D_{k k}\left(x_{k}\right)$

$D_{i i}\left(x_{k} \cdot y_{k}\right)=0$ for $i \neq k$

$D_{i j}\left(x_{k} \cdot y_{k}\right)=x_{k} \cdot D_{i j}\left(y_{k}\right)+y_{k} \cdot D_{i j}\left(x_{k}\right)$ if $i<j$ and $i=k$ or $j=k$, $D_{i j}\left(x_{k} \cdot y_{k}\right)=0$ if $k \neq i$ and $k \neq j$.

In particular, putting $y_{k}=e_{k}, D_{i i}\left(x_{k}\right)=0=D_{i j}\left(x_{k}\right)$ if $k \neq i, k \neq j$.

Since $D_{i i}$ is a derivation of $J_{i}$ into $M_{i i}$, there exist $z_{r} \in J_{i}, m_{r} \in M_{i i}$ such that $D_{i i}\left(x_{i}\right)=\sum_{r}\left(z_{r}, x_{i}, m_{r}\right)$ for $x_{i} \in J_{i}$. Also, $D_{i i}\left(x_{k}\right)=0=\sum_{r}\left(z_{r}, x_{k}, m_{r}\right)$ for $x_{k} \in J_{k}, k \neq i$. Thus $D_{i i}(x)=\sum_{r}\left(z_{r}, x, m_{r}\right)$ for all $x \in J$.

Now let $i \neq j$. Then $D_{i j}$ is a derivation on $J_{i}+J_{j}$ into $M_{i j}$, and is zero on $J_{k}$ for $k \neq i$ or $j$. Since $M_{i j}$ is a unital module for $J_{i}+J_{j}, D_{i j}$ annihilates $e_{i}+e_{j} . \quad D_{i j}\left(e_{i}\right)=-D_{i j}\left(e_{j}\right) . \quad M_{i j}$ is a special module for $J_{i}$, so (as shown in 1.) $D_{i j}\left(x_{i}\right)=4\left(e_{i}, x_{i}, D_{i j}\left(e_{i}\right)\right)=2\left(e_{i}, x_{i}, D_{i j}\left(e_{i}-e_{j}\right)\right)=$ $\left(e_{i}-e_{j}, x_{i}, D_{i j}\left(e_{i}-e_{j}\right)\right)$ for $x_{i} \in J_{i}$, and similarly $D_{i j}\left(x_{j}\right)=4\left(e_{j}, x_{j}, D_{i j}\left(e_{j}\right)\right)=$ $\left(e_{i}-e_{j}, x_{j}, D_{i j}\left(e_{i}-e_{j}\right)\right)$ for $x_{j} \in J_{j}$. Thus $D_{i j}(x)=\left(e_{i}-e_{j}, x, D_{i j}\left(e_{i}-e_{j}\right)\right)$ for $x \in J_{i}+J_{j}$, and $D_{i j}\left(x_{k}\right)=0=\left(e_{i}-e_{j}, x_{k}, D_{i j}\left(e_{i}-e_{j}\right)\right)$ for $k \neq i$ or $j$, so $D_{i j}(x)=\left(e_{i}-e_{j}, x, D_{i j}\left(e_{i}-e_{j}\right)\right)$ for all $x \in J$.

Thus all $D_{i i}$ and $D_{i j}$ satisfy (1), and so $D$ does also.

3. Let $J$ be simple and special, $M$ unital, and the base field algebraically closed. Assume that the degree of $J$ is not divisible by the characteristic, and further that $J$ is not $H\left(Q_{3}\right)$. We may also assume the degree of $J$ is at least two. 
Since $J$ has an identity, the algebra $U^{\prime}$ is the direct sum of an ideal $U_{s}$ and a one dimensional ideal $F e_{0}$, where $U_{s}$ is the ideal in $U^{\prime}$ generated by the identity element $e$ of $J . U_{s}$ is the universal algebra for special representations such that $2 e$ is the identity operator, that is, $2 e$ is the identity element of $U_{s}$. The ideal in $U^{\prime \prime}$ generated by the elements $x \otimes e+e \otimes x$ with $x$ in $J$ is denoted by $U_{s}^{(2)}$ : this is the universal algebra for $U^{\prime \prime}$ representations which are also unital for $J$. From now on we shall consider only $U_{s}$ and $U_{s}^{(2)}$ instead of $U^{\prime}$ and $U^{\prime \prime}$, and denoted by 1 the identity element $2 e$ of $U_{s}$.

For the special simple algebras we are considering (i.e. excluding $H\left(Q_{3}\right)$ ), every unital module is equivalent to a $U_{s}^{(2)}$ module. Further, $U_{s}$ and $U_{s}^{(2)}$ are separable if $J$ is, so all unital module are completely reducible. The irreducible unital modules for each of these algebras are all obtained by decomposing a certain unital module which is also a $U_{s}$ bimodule, and we may assume that the unital module $M$ we are considering is this one in order to show the derivations satisfy (1). Thus in $M, x \cdot m=$ $x m+m x$. Let $D$ be a derivation of $J$ into $M$ and let $B$ be the associative algebra $U_{s} \oplus M$ with multiplication $(a+m)(b+n)=a b+(a n+m b)$. Then the map: $x \rightarrow x+D(x)$ of $J$ into $B$ satisfies

$$
h(x \cdot y)=h(x) h(y)+h(y) h(x),
$$

that is, $h$ is a Jordan homomorphism. Also, $h(e)=e=\frac{1}{2} \cdot$ (identity element of $B$ ). By the definition of $U_{s}, h$ extends to a homomorphism, also denoted by $h$, of $U_{s}$ into $B$. Since $U_{s}$ is generated by $J$ and since $h(x)=x+D(x)$ for $x$ in $J, h(a)=a+d(a)$ for $\alpha$ in $U_{s}$, where $d(a) \in M$. Since $h$ is a homomorphism, $d$ is a derivation.

Since $U_{s}$ is a separable associative algebra, $d(a)=[a, m]$ for some $m \in M$, and $D(x)=[x, m]$ for $x$ in $J$. By (7), we see that $D$ is of the form (1) if $m=\sum_{i}\left[z_{i}, m_{i}\right]$ for elements $z_{i}$ in $J, m_{i}$ in $M$. It is clear that $m$ is determined only modulo $Z(M)=\{n \in M \mid[x, n]=0$ for all $x \in J\}$. Thus we only have to show that $M=Z(M)+[J, M]$, and for this purpose we will examine separately the various types of simple Jordan algebras.

First, let $J=F e+V$ be of degree two. $U_{s}$ is the Clifford algebra of the space $V$ and its inner product, and $M$ may be taken as $U_{s}$ itself if $V$ has even dimension, while if $V$ has odd dimension $M$ is the Clifford algebra of a space $W$ of dimension one larger: $W=V+F u$ where $(u, u)=1,(u, v)=0$ for all $v \in V$. Assume now that $V$ has dimension $r$, and let $x_{1}, \cdots, x_{r}$ be a basis for $V$ satisfying $\left(x_{i}, x_{t}\right)=1,\left(x_{i}, x_{j}\right)=0$ for $i \neq j$. A basis for $M=U_{s}$ consists of 1 and all monomials

$$
x_{i_{1}} x_{i_{2}} \cdots x_{i_{k}}, i_{1}<i_{2}<\cdots<i_{k} .
$$

$Z(M)$ is just $F 1$. We have $x_{i} x_{j}=-x_{j} x_{i}$ for $i \neq j, x_{i}^{2}=1$, so 


$$
\begin{aligned}
\frac{1}{2}\left[x_{i_{1}}, x_{i_{1}} x_{i_{2}} \cdots x_{i_{k}}\right] & =\frac{1}{2}\left(1-(-1)^{k-1}\right) x_{i_{2}} \cdots x_{i_{k}} \\
& =x_{i_{2}} \cdots x_{i_{k}} \text { if } k-\text { is odd } ; \\
\frac{1}{2}\left[x_{i_{1}}, x_{i_{2}} \cdots x_{i_{k}}\right] & =\frac{1}{2}\left(1-(-1)^{k-1}\right) x_{i_{1}} \cdots x_{i_{k}} \\
& =x_{i_{1}} \cdots x_{i_{k}} \text { if } k \text { is even. }
\end{aligned}
$$

Thus every $x_{i_{1}} \cdots x_{i_{k}} \in[J, M]$.

Now let $V$ have add dimension $r(\geqq 3)$, and choose a basis $x_{1}, \cdots, x_{r}$ for $V$ satisfying the same conditions as above, so $\left\{x_{1}, \cdots, x_{r}, u\right\}$ is an orthonormal basis for $W$. By the above calculations $[J, M]$ contains $x_{i_{1}} \cdots x_{i_{k}}$ if $k<r$ and also contains $u$ and $x_{i_{1}} \cdots x_{i_{k}} u$ for all $k$. The only term left is $x_{1} \cdots x_{r}$, but this is clearly in $Z(M)$. Thus $M=$ $Z(M)+[J, M]$.

Next we consider the simple Jordan algebra $H\left(F_{n}\right), n \geqq 3$, of $n \times n$ symmetric matrices over the base field $F . \quad U_{s}$ is $F_{n}$, and $M$ may be taken as $F_{n}$ also ([7], Prop. 19.2). Since $F_{n}$ is the enveloping algebra of $H\left(F_{n}\right)$, the set of elements in $M$ that commute with those of $J$ is just the center $F 1$ of $F_{n}$, and we have to show $F_{n}=F \cdot 1+\left[J, F_{n}\right]$ under the assumption that the characteristic $p$ does not divide $n$. Let $e_{i j}$ denote the matrix units in $F_{n}$. Then $\left[e_{i i}, e_{i j}\right]=e_{i j} \in\left[J, F_{n}\right]$ for $i \neq j$. Also $\left[e_{i j}+e_{j i}, \alpha e_{j i}\right]=\alpha\left(e_{i i}-e_{j j}\right)$ for $\alpha \in F$. Now let $x=\sum \alpha_{i j} e_{i j}$ be a matrix of trace zero: $\sum_{i} \alpha_{i i}=0$; then $x=\sum_{i \neq j} \alpha_{i j} e_{i j}+\sum_{i \neq 1} \alpha_{i i}\left(e_{i i}-e_{11}\right)$ since $\alpha_{11}=-\sum_{i \neq 1} \alpha_{i i}$ so $x \in[J, M]$. Finally, for arbitrary $y=\sum \beta_{i j} e_{i j}$, if $\tau=$ trace of $y=\sum_{i} \beta_{i i}$, let $x=y-\frac{1}{n} \tau 1$ (1 being the identity matrix). Then $x$ has trace zero, so $\varepsilon[J, M]$, and $y=\frac{1}{n} \tau 1+x \in F 1+[J, M]$. We note that if $p$ divides $n$, then every element in $F 1+[J, M]$ has trace zero so $M \neq F 1+[J, M]$.

Now let $E$ be the two dimensional algebra over $F$ and $J=H\left(E_{n}\right)$. Then $U_{s}=E_{n}$ and $M$ may be taken as $Q_{n}(Q$ being the quaternion algebra): this is shown in the proof of [7], Prop. 19.4. Since $E_{n}=$ $E \otimes F_{n}, Q_{n}=Q \otimes F_{n}$, and the centralizer of $E$ in $Q$ is $E$, the centralizer of $E_{n}$ (and also of $J$ ) in $Q_{n}$ is $E \cdot 1=\{\alpha 1 / \alpha \in E\}$. For any $q \in Q, q e_{i j}=$ $\left[e_{i i}, q e_{i j}\right] \in[J, M]$, and $q e_{i i}-q e_{j j}=\left[e_{i j}+e_{j i}, q e_{j i}\right] \in[J, M]$. Let $r \in E, q \in Q$, then $\left[r e_{i j}+\bar{r} e_{j i}, q e_{j i}\right]=r q e_{i i}-q r e_{j j}=r q\left(e_{i i}-e_{j j}\right)+[r, q] e_{j j}$ for $i \neq j$. Since $r q\left(e_{i i}-e_{j j}\right) \in[J, M],[r, q] e_{j j} \in[J, M]$ also, so $[E, Q] 1 \in[J, M]$. From these calculations it follows that if $x=\sum q_{i j} e_{i j}$ and $\tau=\sum_{i} q_{i i}$ then $\frac{1}{n} \tau 1 \in[J, M]$. Also $Q=E+[E, Q]$ and $[E, Q] 1 \in[J, M]$ so $\frac{1}{n} \tau=$ $c+d, c \in[J, M], d \in E$, and $x \in[J, M]+E 1$, which we had to show.

Lastly we have to consider $J=H\left(Q_{n}\right)$. (We are assuming $n \neq 3$, but everything we say is valid for $n=3$ also provided the module $M$ 
is assumed to be a $U_{s}^{(2)}$ module. $H\left(Q_{3}\right)$ has one other type of unital module which we will consider later.) Here $U_{s}=Q_{n}=M$, the center of $M$ is $F 1$, and $M=F 1+[J, M]$ if $p$ does not divide $n$ : the proof is the same as above.

4. We have left the simple Jordan algebras $H\left(C_{3}\right)$ and $H\left(Q_{3}\right)$ but before considering these we have to prove Theorem 3 for the alternative algebras $C$ and $Q$. In fact, to prove Theorem 3 for any separable alternative algebra over any base field we need only prove it when: (a) the base field is algebraically closed (this is easy to see because of the forms (11), (12) for inner derivations); (b) the algebra is simple and the bimodule is unital ([13]); (c) the algebra is either $C$ or $Q$ (since, as shown in [7], all other simple alternative algebras and bimodules are actually associative algebras and associative bimodules).

If $A$ is any alternative algebra with identity and $N$ a unital alternative bimodule, then on introducing the product $a \cdot b$ for $a \in A, b \in A$, or $\in N$, we obtain a special Jordan algebra $(A, \cdot)$ and a $U^{\prime \prime}$ module $(N, \cdot)$ for $(A, \cdot)$.

From now on, let $A$ denote either $C$ or $Q, N$ a unital alternative bimodule, and assume the base field algebraically closed. $(A, \cdot)$ is then a simple Jordan algebra of degree two: in fact $A$ has an involution $a \rightarrow \bar{a}$, and $(A, \cdot)=F 1+V$ where $V=A^{-}=\{a \in A \mid \bar{a}=-a\}$ and for $a, b \in A^{-}$we set $(a, b)=a \cdot b=a b+b a . F 1$ is the center of $A$, and the nucleus of $A$ if $A=C$. If $D$ is a derivation of $A$ into $N$, it is also a derivation of $(A, \cdot)$ into $(N, \cdot)$, and by our results for Jordan algebras of degree two,

$$
\begin{aligned}
D(a) & =\sum_{i}\left(x_{i} \cdot a\right) \cdot m_{i}-x_{i} \cdot\left(a \cdot m_{i}\right) \\
& =\sum_{i} a\left[R_{x_{i}}+L_{x_{i}}, R_{m_{i}}+L_{m_{i}}\right]
\end{aligned}
$$

where $x_{i} \in A, m_{i} \in N$, and $a R_{x}=a x, a L_{x}=x a$.

(If $A=C$, then $N$ is a direct sum of copies of $C$, and may be taken as $C$ also, and it is possible to give an even shorter proof of (13) in this case: the derivation $D$ maps $C^{-}$into itself since $C^{-}=\{(a, b, c)=$ $(a \cdot b) \cdot c-a \cdot(b \cdot c) \mid a, b, c \in C\}$. Also, $D(\alpha 1)=0$ for $\alpha \in F$, so for $x, y \in C^{-}, D(x \cdot y)=0=D(x) \cdot y+x \cdot D(y)$, or $(D(x), y)+(x, D(y))=0$ where $(x, y)=(x \cdot y) 1$. Thus $D$ is a skew linear transformation in $C^{-}$, and so, relative to an orthonormal basis $\left\{x_{i}\right\}$ for $C^{-}$,

$$
\left.D(x)=\sum_{i<j} \alpha_{i j}\left(\left(x, x_{i}\right) x_{j}-\left(x, x_{j}\right) x_{i}\right)=\sum_{i<j} \alpha_{i j}\left(x_{i}, x, x_{j}\right) .\right)
$$

Since $D$ satisfies (13), it is inner in the sense that it belongs to the Lie algebra generated by the right and left multiplications. However, we want to show that $D$ has the specific form (11). 
Let $D_{x, z}=\left[R_{x}, R_{z}\right]+\left[L_{x}, R_{z}\right]+\left[L_{x}, L_{z}\right]$ where all the operators act on the right of elements. Then $a \rightarrow a D_{x, z}$ is a derivation for all $x, z$, and the following identities are valid for characteristic not 2 [(12], formulas (6), (12), (19)):

$$
\begin{gathered}
{\left[L_{x}, R_{z}\right]=\left[R_{x}, L_{z}\right]} \\
D_{x, z}=R_{[x, z]}-L_{[x, z]}-3\left[L_{x}, R_{z}\right] \\
{\left[R_{x}+L_{x}, R_{z}+L_{z}\right]=D_{x, z}+\left[L_{x}, R_{z}\right]} \\
=R_{[x, z]}-L_{[x, z]}-2\left[L_{x}, R_{z}\right] \\
{\left[x, y_{1} y_{2}, z\right]=y_{1}\left[x, y_{2}, z\right]+\left[x, y_{1}, z\right] y_{2}+\left[([x, z]), y_{1}, y_{2}\right] .}
\end{gathered}
$$

Now take $A=C$, and $N=C$ (which we may do, as remarked above). Let $D$ be a derivation satisfying (13). Since $C=F 1+C^{-}$, we may assume the $x_{i}$ and $m_{i}$ are in $C^{-}$.

Assume first that the characteristic is not 3. For $y$ in $C$,

$$
\begin{aligned}
D(y) & =\sum_{i} y\left[R_{x_{i}}+L_{x_{i}}, R_{m_{i}}+L_{m_{i}}\right] \\
& =\sum_{i} y\left(D_{x_{i}, m_{i}}+\left[L_{x_{i}}, R_{m_{i}}\right]\right) \\
& =\frac{1}{3} \sum_{i} y\left(R_{\left[x_{i}, m_{i}\right]}-L_{\left[x_{i}, m_{i}\right]}+2 D_{x_{i}, m_{i}}\right) .
\end{aligned}
$$

Since $\frac{2}{3} \sum_{i} D_{x_{i}, m_{i}}$ is a derivation, $\sum_{i} R_{\left[x_{i}, m_{i}\right]}-L_{\left[x_{i}, m_{i}\right]}$ is also a derivation, and by Lemma 1 of [12], $\sum_{i}\left[x_{i}, m_{i}\right]$ is in the nucleus of $C$. However, since the $x_{i}$ and $m_{i}$ are in $C^{-}, \sum_{i}\left[x_{i}, m_{i}\right]$ is also in $C^{-}$, and so $\sum\left[x_{i}, m_{i}\right]=0$. Thus by (15),

$$
\begin{aligned}
D(y) & =\frac{2}{3} y \sum_{i} D_{x_{i}, m_{i}}=-2 \sum_{i} y\left[L_{x_{i}}, R_{m_{i}}\right] \\
& =\sum_{i}\left[-2 x_{i}, y, m_{i}\right]
\end{aligned}
$$

and so $D$ has form (11) with $g=0$.

Now let the characteristic be 3 , and $D$ a derivation in $C$ satisfying (13). By (15), for all $x, z, D_{x, z}=R_{[x, z]}-L_{[x, z]}$. Since

$$
D=\sum_{i}\left[R_{x_{i}}+L_{x_{i}}, R_{m_{i}}+L_{m_{i}}\right]=\sum_{i} D_{x_{i}, m_{i}}+\left[L_{x_{i}}, R_{m_{i}}\right]
$$

is a derivation, $\sum_{i}\left[L_{x_{i}}, R_{m_{i}}\right]$ is also a derivation, that is, $\sum_{i}\left[x_{i}, y z, m_{i}\right]=$ $y \sum_{i}\left[x_{i}, z, m_{i}\right]+\sum_{i}\left[x_{i}, y, m_{i}\right] z$ for all $y, z$ in $C$. By $(17),\left[y, z,\left(\sum_{i}\left[x_{i} m_{i}\right]\right)\right]=$ 0 for all $y, z$, so $\sum\left[x_{i}, m_{i}\right]$ is in the nucleus of $C$. Since it is also in $C^{-}, \sum_{i}\left[x_{i}, m_{i}\right]=0$. Thus $\sum_{i} D_{x_{i}, m_{i}}=0$ and $D=\sum_{i}\left[L_{x_{i}}, R_{m_{i}}\right]$, that is, $D(y)=\sum_{i}\left[x_{i}, y, m_{i}\right]$.

Next we have to consider the derivations of $Q$. The irreducible 
unital alternative bimodules for $Q$ are $Q$ itself, and a certain submodule of $Q v$, where $C=Q+Q v$ : from (10) it follows that if $I$ is any irreducible (therefore two-dimensional) right ideal in $Q$, then $I v$ is a unital irreducible bimodule for $Q$ (called a Cayley bimodule in [7]). Thus we have only to consider derivations of $Q$ into $I v$, but since $Q v$ is a direct sum of two submodules isomorphic to $I v$, we may take the module to be $Q v$. The proof is almost the same as for $C$. If $D$ satisfies (13), we may take the $x_{i}$ in $Q^{-}=Q \cap C^{-}$; the $m_{i}$ are automatically in $C^{-}$. The nucleus of $Q v$ is zero, as shown by (10). As for $C$, we conclude that $\sum_{i}\left[x_{i}, m_{i}\right]=0$, and so $D$ satisfies (11), and (12) for characteristic not 3 . This concludes the proof of Theorem 3 .

5. We can now apply the results of 4 to $H\left(C_{3}\right)$ and $H\left(Q_{3}\right)$, and we start with $H\left(C_{3}\right)$.

If $J=H\left(C_{3}\right)$, the only irreducible unital module for $J$ is $J$ itself. Let $J=H\left(C_{3}\right), M$ be isomorphic to $H\left(C_{3}\right)$, and $E=J+M$, the split null extension. $E$ may be regarded as a subspace closed under $x \cdot y=$ $x y+y x$ of the nonassociative algebra $B=A \oplus N$, where $A$ and $N$ are both isomorphic to $C_{3}$, and $N$ is an $A$-bimodule in the sense that $a n, n a \in N$ for $a \in A, n \in N . \quad J, M$, and $E$ are the self-adjoint elements of $A, N, B$, respectively (see $\S 10$ of [7]).

Let $K=H\left(F_{3}\right)$ be the subalgebra of $J$ of matrices with coefficients in $F$, and $L=F_{3}$ the subalgebra of $A$ of matrices with coefficients in $F$, so $K=L \cap J$. K, J, L are all contained in $A$. $L$ is in the nucleus of $B$ (elements of $L$ associate with all elements of $B$ ), and $B$ and $N$ are associative bimodules for $L$.

Let $D$ be a derivation of $J$ into $M$. Let $h(x)=x+D(x)$ for $x \in J$. Then $h(x \cdot y)=h(x) \cdot h(y)$ for $x, y \in J$. We want to show first that $h$ can be extended from $K=J \cap L$ to be a homomorphism of $L$ into $B$ (that is, $h\left(a_{1} a_{2}\right)=h\left(a_{1}\right) h\left(a_{2}\right)$ for $a_{i} \in L$ ), and this will follow from Theorem 7.3 of [7] if we show that $h(K)$ generates an associative subalgebra in $B$. For $x \in K, h(x)=x+D(x) \in L+N$, and $L+N$ is an associative subalgebra of $B$ which contains the subalgebra generated by $h(K)$.

Thus $h$ can be extended from $K$ to a homomorphism of $L$ into $B$. Since $K$ generates $L$ and $h(x)=x+D(x)$ for $x \in K, h(a)=a+d(a)$ for $a$ in $L$, where $d$ is a derivation of $L$ into $N$ such that $d(x)=D(x)$ for $x$ in $K$. Since $N$ is an associative bimodule for $L, d(a)=[a, n]$ for a skew element $n$ of $N$. However, every skew element of $C_{3}$ is a sum of commutators of hermitian elements (by Prop. 7.3 of [7], as $C^{-}=$ $\left.\left[C^{-}, C^{-}\right]\right)$, so $n=\sum_{i}\left[z_{i}, m_{i}\right], z_{i} \in M=H(N)$, and so $D(x)=\sum_{i}\left[x,\left[z_{i} m_{i}\right]\right]=$ $\sum\left(z_{i}, x, m_{i}\right)$ for $x \in K$.

For any $y$ in $J$, the map $D_{1}(y)=D(y)-\sum_{i}\left(z_{i}, y, m_{i}\right)$ is a derivation which annihilates $K$, and it suffices to show $D_{1}$ is inner. Thus we 
may assume $D$ is zero on $K$, and so $h(x)=x$ for $x \in K$, where $h(x)=$ $x+D(x)$ for $x \in D$. Thus $h(K)=K \subseteq L$, so $h(K)$ is in the nucleus of $B$, and, using Th. 7.3 of [7] again, $h$ can be extended from $J$ to a homomorphism of $A=C_{3}$ into $B$. Since $J$ generates $A, h(a)=a+d(a)$ for $a \in A$, and $d$ is a derivation of $A$ into $N$. Since $d(x)=D(x)$ for $x \in J$, we will write $D$ for $d$.

Let $a=\sum_{i, j} \alpha_{i j} e_{i j}$ be an element of $A, \alpha_{i j}$ being in $C$. If $I$ denotes the identity matrix, the elements $\alpha I, \alpha \in C$, form a subalgebra of $A$ isomorphic to $C$, and we will denote this subalgebra by $C I$, so $a=$ $\sum_{i, j}\left(\alpha_{i j} I\right) e_{i j}, \alpha_{i j} I \in C I$.

Since $D\left(e_{i j}\right)=0, D(\alpha)=\sum_{i, j} D\left(\alpha_{i j} I\right) e_{i j}$. For $\alpha \in C,\left[\alpha I, e_{i j}\right]=0$ for all $i, j$, so $\left[D(\alpha I), e_{i j}\right]=0$ and $D(\alpha I)=\alpha^{\prime} I, \alpha^{\prime} \in C$. The map $\alpha I \rightarrow \alpha^{\prime} I$ is clearly a derivation of $C I$ into the bimodule $N_{0}=\left\{n \in N \mid\left[e_{i j}, n\right]=0\right.$ for all $i, j\}$ which is clearly isomorphic to $C I$ itself as $C I$ bimodule. Thus $\alpha^{\prime}=\sum_{i}\left[\sigma_{i}, \alpha, \tau_{i}\right]$ for elements $\sigma_{i}, \tau_{i} \in C^{-}$such that $\sum\left[\sigma_{i}, \tau_{i}\right]=0$.

If $a=\sum \alpha_{i j} e_{i j} \in J, D(a)=\sum \alpha_{i j}^{\prime} e_{i j}$. We will show $D(\alpha)$ satisfies (1) by proving this for elements $a=\alpha e_{12}+\bar{\alpha} e_{21}$ and for the elements of $K$ and noting that $J$ is generated by such elements.

From now on we identify $J$ with $M$. We note that the derivation $\alpha \rightarrow \alpha^{\prime}$ in $C$ satisfies $(\alpha)^{\prime}=\left(\bar{\alpha}^{\prime}\right)$ since this holds for $\alpha \in C^{-}$and for $\alpha \in F 1$. Let $b_{i}=\sigma_{i} e_{12}+\bar{\sigma}_{i} e_{21}, c_{i}=\tau_{i} e_{12}+\bar{\tau}_{i} e_{21}$. Then

$$
\begin{aligned}
\left(b_{i}, a, c_{i}\right) & =\left(b_{i} \cdot a\right) \cdot c_{i}-b_{i} \cdot\left(a \cdot c_{i}\right) \\
& =\left(\sigma_{i} e_{12}+\bar{\sigma}_{i} e_{21}, \alpha e_{12}+\bar{\alpha} e_{21}, \tau_{i} e_{12}+\bar{\tau}_{i} e_{21}\right) \\
& =z_{i} e_{12}+\bar{z}_{i} e_{21}
\end{aligned}
$$

where $z_{i}=\left(\sigma_{i} \bar{\alpha}\right) \tau_{i}+\left(\alpha \bar{\sigma}_{i}\right) \tau_{i}+\tau_{i}\left(\bar{\sigma}_{i} \alpha\right)+\tau_{i}\left(\bar{\alpha} \sigma_{i}\right)-\left(\tau_{i} \bar{\alpha}\right) \sigma_{i}-\left(\alpha \bar{\tau}_{i}\right) \sigma_{i}-\sigma_{i}\left(\bar{\tau}_{i} \alpha\right)$ $-\sigma_{i}\left(\bar{\alpha} \tau_{i}\right)$.

But $\bar{\sigma}_{i}=-\sigma_{i}, \bar{\tau}_{i}=-\tau_{i}$, so

$$
\begin{aligned}
z_{i}= & \left(\sigma_{i} \bar{\alpha}\right) \tau_{i}-\left(\alpha \sigma_{i}\right) \tau_{i}-\tau_{i}\left(\sigma_{i} \alpha\right)+\tau_{i}\left(\bar{\alpha} \sigma_{i}\right) \\
& -\left(\tau_{i} \bar{\alpha}\right) \sigma_{i}+\left(\alpha \tau_{i}\right) \sigma_{i}+\sigma_{i}\left(\tau_{i} \alpha\right)-\sigma_{i}\left(\bar{\alpha} \tau_{i}\right) \\
= & {\left[\sigma_{i}, \bar{\alpha}, \tau_{i}\right]-\left[\tau_{i}, \bar{\alpha}, \sigma_{i}\right]-\alpha\left(\sigma_{i} \tau_{i}\right)-\left[\alpha, \sigma_{i}, \tau_{i}\right]-\left(\tau_{i} \sigma_{i}\right) \alpha } \\
& +\left[\tau_{i}, \sigma_{i}, \alpha\right]+\alpha\left(\tau_{i} \sigma_{i}\right)+\left[\alpha, \tau_{i}, \sigma_{i}\right]+\left(\sigma_{i} \tau_{i}\right) \alpha-\left[\sigma_{i}, \tau_{i}, \alpha\right] \\
= & 2\left[\sigma_{i}, \bar{\alpha}, \tau_{i}\right]+4\left[\sigma_{i}, \alpha, \tau_{i}\right]+\left[\alpha,\left[\tau_{i}, \sigma_{i}\right]\right] .
\end{aligned}
$$

For any $\beta$ in $C, \beta+\bar{\beta}$ is in the center and nucleus, so for every $\gamma, \delta,[\gamma, \beta+\bar{\beta}, \delta]=0$ or $[\gamma, \beta, \delta]=-[\gamma, \bar{\beta}, \delta]$; also $[\bar{\gamma}, \bar{\beta}, \bar{\delta}]=-[\bar{\delta}, \bar{\beta}, \bar{\gamma}]=$ $[\bar{\gamma}, \bar{\beta}, \bar{\delta}]=[\gamma, \bar{\beta}, \delta]$. Thus $z_{i}=2\left[\sigma_{i}, \alpha, \tau_{i}\right]+\left[\alpha,\left[\tau_{i}, \sigma_{i}\right]\right]$. By assumption $\sum_{i}\left[\sigma_{i}, \tau_{i}\right]=0$, so, finally, $\sum_{i}\left(b_{i}, a, c_{i}\right)=2\left(\sum_{i}\left[\sigma_{i}, \alpha, \tau_{i}\right] e_{12}+\left[\sigma_{i}, \bar{\alpha}, \tau_{i}\right] e_{21}\right)$ and $D(a)=\frac{1}{2} \sum_{i}\left(b_{i}, a, c_{i}\right)$ for $a=\alpha e_{12}+\bar{\alpha} e_{21}$. For $x \in H\left(F_{3}\right), \sum_{i}\left(b_{i}, x, c_{i}\right)=$ $\sum_{i}\left[x,\left[b_{i}, c_{i}\right]\right]$ since $x$ is in the nucleus of $C_{3}$, and 


$$
\sum_{i}\left[b_{i}, c_{i}\right]=\sum_{i}\left(\sigma_{i} \bar{\tau}_{i}-\tau_{i} \bar{\sigma}_{i}\right) e_{11}+\left(\bar{\sigma}_{i} \tau_{i}-\bar{\tau}_{i} \sigma_{i}\right) e_{22}=\sum_{i}\left[\tau_{i}, \sigma_{i}\right]\left(e_{11}+{ }_{22}\right)=0
$$

since $\sum_{i}\left[\sigma_{i}, \tau_{i}\right]=0$. Thus $D(x)=\frac{1}{2} \sum\left(b_{i}, x, c_{i}\right)=0$ for $x \in H\left(F_{3}\right)$. It is easy to see that $H\left(F_{3}\right)$ and elements $\alpha$ of the form $\alpha e_{12}+\bar{\alpha} e_{21}$ generate $J$, and so $D(z)=\frac{1}{2} \sum_{i}\left(b_{i}, z, c_{i}\right)$ for all $z \in J$.

Now we have to consider derivations of $H\left(Q_{3}\right)$ into the module $H\left((Q v)_{3}\right)$ consisting of hermitian matrices with elements in the subspace $Q v$ of $C$. Since $H\left(C_{3}\right)$ is the direct sum, as $H\left(Q_{3}\right)$ module, of the submodules $H\left(Q_{3}\right)$ and $H\left((Q v)_{3}\right)$ it suffices to show that a derivation of $H\left(Q_{3}\right)$ into $H\left(C_{3}\right)$ has the form (1), and this can be done exactly as for derivation of $H\left(C_{3}\right)$ into itself; we shall therefore omit the proof. (The result is valid even in characteristic 3 , but in characteristic 3 there exist other unital modules for $H\left(Q_{3}\right)$ (for example, $Q_{3}$ ) for which not all derivations satisfy (1); we will show this below.)

(b) We have shown that if $J$ is any semi-simple Jordan algebra over an algebraically closed field and none of the special simple ideals of $J$ has degree divisible by the characteristic of the field, then every derivation of $J$ is of the form (1). Suppose now that $J$ is separable over an arbitrary base field, and that the special simple ideals of $J$ satisfy the same condition on degrees (i.e. when their centers are extended to their algebraic closures, the maximum number of orthogonal idempotents with sum 1 is not divisible by the characteristic). Then, when the base field is extended to its algebraic closure, $J$ will still be semi-simple and satisfy the degree condition, for if the base field of a simple separable Jordan algebra is extended to its algebraic closure, it becomes a direct sum of isomorphic simple algebras which are also isomorphic to the algebra obtained by extending the center of the simple algebra. Extension of the base field also preserves the property of being special. A derivation of $J$ over the original base field into a module extends by linearity to a derivation of the algebra obtained by extending the base field into the module obtained by extending the base field, and it is easy to see that the latter derivation is of the form (1) if and only if the former is.

(c) To complete the proof of the theorem, we have to show that if $J$ is separable but does not satisfy the condition on degrees, there exist derivations not of the form (1). Let $J_{1}$ be an ideal of $J$ which is simple, separable and special and of degree divisible by the characteristic; it suffices to find a module and a derivation for $J_{1}$ which do not satisfy (1), i.e. we may assume $J$ is $J_{1}$, since the module and derivation for $J_{1}$ may be considered as module and derivation for $J$ in the obvious way. Let $J_{E}$ denote $J \otimes_{F} E$ for an extension field $E$ of $F$. Then $U_{s}\left(J_{E}\right)=$ $U_{s}(J)_{E}$. We take $U_{s}$ as the module. If there is an element $m \in U_{s}$ such that $m \notin$ Center of $U_{s}+\left[J, U_{s}\right]$ then the derivation $x \rightarrow[x, m]$ will do. 
In particular, it will suffice to find $m \notin$ Center of $U_{s}+\left[U_{s}, U_{s}\right]$. However, (Center of $\left.U_{s}\right)_{E}=$ Center of $\left(U_{s}\right)_{E}$, and $\left(\left[U_{s}, U_{s}\right]\right)_{E}=\left[\left(U_{s}\right)_{E},\left(U_{s}\right)_{E}\right]$, so it sufficies to show that $U_{s}\left(J_{E}\right) \neq$ Center of $U_{s}\left(J_{E}\right)+\left[U_{s}\left(J_{E}\right), U_{s}\left(J_{E}\right)\right]$ for $E$ the algebraic closure of $F$.

$J_{E}$ is a direct sum of isomorphic simple algebras $\left(J_{E}\right)_{i}$ and $U_{s}\left(J_{E}\right)$ is the direct sum of the algebras $U_{s}\left(\left(J_{E}\right)_{i}\right)$, so finally it suffices to show that $U_{s} \neq$ Center $+\left[U_{s}, U_{s}\right]$ if $J$ is simple over an algebraically closed field and of degree $n$ divisible by the characteristic. But then $U_{s}=D_{n}$ where $D=F, E$, or $Q$, and in each of these matrix algebras the matrix $e_{11} \notin$ Center $+\left[U_{s}, U_{s}\right]$ if $p$ divides $n$.

\section{REFERENCES}

1. A. A. Albert, A structure theory for Jordan algebras, Ann. of Math., 48 (1947), 446-467.

2. —— A theory of power associative commutative algebras, Trans. Amer. Math. Soc. 69 (1950), 503-527.

3. H. E. Campbell, On the Casimir operator, Pacific J. Math. 7 (1957) 1325-1331.

4. H. Cartan and S. Eilenberg, Homological Algebra, Princeton, 1956.

5. N. Jacobson, Derivation algebras and multiplicalion algebras of semi-simple Jordan algebras, Ann. of Math., 50 (1949), 866-874.

6. - General representation theory of Jordan algebras, Trans. Amer. Math. Soc., 70 (1951), 509-530.

7. _- Siructure of alternative and Jordan bimodules, Osaka Math. J., 6 (1954), $1-71$.

8. $\quad$, A theorem on the structure of Jordan algebras, Proc. Nat. Acad. Sci., 42 (1956), $140-147$.

9. - Jordan algebras, Report of a Conference on Linear Algebras, National Academy of Sciences Publication 502, 1957.

10. I. Kaplansky, Seminar on simple Lie algebras, Bull. Amer Math. Soc., 60 (1954), 471.

11. R. D. Schafer Inner derivations of non-associative algebras, Bull. Amer. Math. Soc., 55 (1949), 769-776.

12. , Representations of alternative algebras, Trans. Amer. Math. Soc. 72 (1957), $1-17$.

13. E. J. Taft. The Whitehead first lemma for alternative algebras, Proc. Amer. Math. Poc. 8 (1957), 950-956.

NORTHWESTERN UNIVERSTTY 


\section{PACIFIC JOURNAL OF MATHEMATICS}

\section{EDITORS}

\section{David Gilbarg}

Stanford University

Stanford, California

\section{R. A. Beaumont}

University of Washington

Seattle 5 , Washington

\author{
A. L. Whiteman
}

University of Southern California Los Angeles 7, California

L. J. Paige

University of California

Los Angeles 24, California

\author{
E. F. BECKENBACH \\ C. E. BURGESS \\ E. HEWITT \\ A. HORN
}

\author{
V. GANAPATHY IYER \\ R. D. JAMES \\ M. S. KNEBELMAN \\ L. NACHBIN
}

ASSOCIATE EDITORS
I. NIVEN

T. G. OSTROM

H. L. ROYDEN

M. M. SCHIFFER
E. G. STRAUS

G. SZEKERES

F. WOLF

K. YOSIDA

\section{SUPPORTING INSTITUTIONS}

\author{
UNIVERSITY OF BRITISH COLUMBIA \\ CALIFORNIA INSTITUTE OF TECHNOLOGY \\ UNIVERSITY OF CALIFORNIA \\ MONTANA STATE UNIVERSITY \\ UNIVERSITY OF NEVADA \\ OREGON STATE COLLEGE \\ UNIVERSITY OF OREGON \\ OSAKA UNIVERSITY \\ UNIVERSITY OF SOUTHERN CALIFORNIA
}

\author{
STANFORD UNIVERSITY \\ UNIVERSITY OF TOKYO \\ UNIVERSITY OF UTAH \\ WASHINGTON STATE COLLEGE \\ UNIVERSITY OF WASHINGTON \\ * * * \\ AMERICAN MATHEMATICAL SOCIETY \\ CALIFORNIA RESEARCH CORPORATION \\ HUGHES AIRCRAFT COMPANY \\ SPACE TECHNOLOGY LABORATORIES
}

Mathematical papers intended for publication in the Pacific Journal of Mathematics should be typewritten (double spaced), and the author should keep a complete copy. Manuscripts may be sent to any one of the four editors. All other communications to the editors should be addressed to the managing editor, L. J. Paige at the University of California, Los Angeles 24, California.

50 reprints per author of each article are furnished free of charge; additional copies may be obtained at cost in multiples of 50 .

The Pacific Journal of Mathematics is published quarterly, in March, June, September, and December. The price per volume (4 numbers) is $\$ 12.00$; single issues, $\$ 3.50$. Back numbers are available. Special price to individual faculty members of supporting institutions and to individual members of the American Mathematical Society: $\$ 4.00$ per volume; single issues, $\$ 1.25$.

Subscriptions, orders for back numbers, and changes of address should be sent to Pacific Journal of Mathematics, 2120 Oxford Street, Berkeley 4, California.

Printed at Kokusai Bunken Insatsusha (International Academic Printing Co., Ltd.), No. 6, 2-chome, Fujimi-cho, Chiyoda-ku, Tokyo, Japan.

PUBLISHED BY PACIFIC JOURNAL OF MATHEMATICS, A NON-PROFIT CORPORATION

The Supporting Institutions listed above contribute to the cost of publication of this Journal, but they are not owners or publishers and have no responsibility for its content or policies. 


\section{Pacific Journal of Mathematics}

\section{Vol. 9, No. $2 \quad$ June, 1959}

Lee William Anderson, On the breadth and co-dimension of a topological lattice

Frank W. Anderson and Robert L. Blair, Characterizations of certain lattices

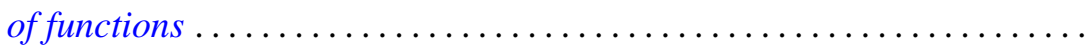

Donald Charles Benson, Extensions of a theorem of Loewner on integral

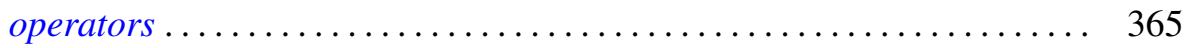

Errett Albert Bishop, A duality theorem for an arbitrary operator ........ 379

Robert McCallum Blumenthal and Ronald Kay Getoor, The asymptotic distribution of the eigenvalues for a class of Markov operators ........

Delmar L. Boyer and Elbert A. Walker, Almost locally pure Abelian

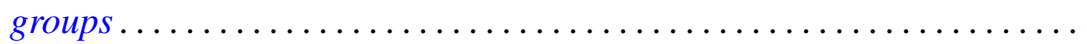

Paul Civin and Bertram Yood, Involutions on Banach algebras ........... Lincoln Kearney Durst, Exceptional real Lehmer sequences .... 415

Eldon Dyer and Allen Lowell Shields, Connectivity of topological

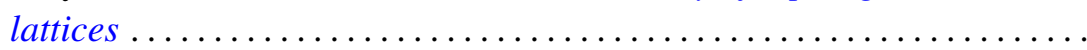

Ronald Kay Getoor, Markov operators and their associated

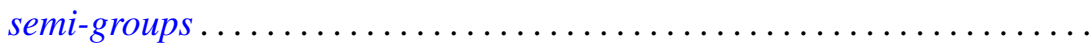

Bernard Greenspan, A bound for the orders of the components of a system of algebraic difference equations

Branko Grünbaum, On some covering and intersection properties in

Minkowski spaces ............................

Bruno Harris, Derivations of Jordan algebras ..............

Henry Berge Helson, Conjugate series in several variables.

Isidore Isaac Hirschman, Jr., A maximal problem in harmonic analysis.

II .

Alfred Horn and Robert Steinberg, Eigenvalues of the unitary part of a matrix

Edith Hirsch Luchins, On strictly semi-simple Banach algebras ...

William D. Munro, Some iterative methods for determining zeros of

functions of a complex variable...

John Rainwater, Spaces whose finest uniformity is metric .

William T. Reid, Variational aspects of generalized convex functions ....

A. Sade, Isomorphisme d'hypergroupoï des isotopes ...... . .

Isadore Manual Singer, The geometric interpretation of a special

connection . . .

Charles Andrew Swanson, Asymptotic perturbation series for characteristic

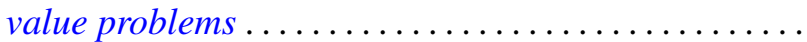

